\title{
Influence of replacing tuberculin skin test with ex vivo interferon $\gamma$ release assays on decision to administer prophylactic antituberculosis antibiotics before anti-TNF therapy
}

- Additional tables are

published online only. To view the files please visit the journal online (http://ard.bmj.com/content/ early/recent)

1Rhumatologie, Hôpital Bicêtre, Assistance Publique-Hôpitaux de Paris (AP-HP), Université Paris-Sud 11, INSERM U1012, 78 rue du Général Leclerc, Le Kremlin Bicêtre, France

${ }^{2}$ Centre d'épidémiologie clinique, Groupe Hospitalier Cochin-Hotel Dieu, AP-HP, Université Paris

Descartes, PRES Sorbonne Paris Cité, INSERM UMR-S 738, Paris, France

320épartement d'Epidémiologie, Hôpital Bichat-Claude Bernard, Biostatistique et Recherche Clinique, Paris, France ${ }^{4}$ Rheumatology and INSERM UMR-S606, Paris Diderot

University, Paris, France

${ }^{5}$ Rhumatologie, Hôpital

Lapeyronie, Université

Montpellier I, Montpellier, France

${ }^{6}$ Rhumatologie, Hôpital Roger

Salengro, Université, Lille, France

${ }^{7}$ Rhumatologie, CHRU de Tours,

Université François Rabelais, CIC INSERM-202, Tours, France

${ }^{8}$ Gastro-entérologie, Hôpital

Saint-Louis, Paris, France

${ }^{9}$ Médecine interne et maladies infectieuses, Hôpital Cochin,

Paris, France

${ }^{10}$ INSERM U996, Microbiologie-

Immunologie Biologique,

Hôpital Antoine Béclère, AP-HP

Université Paris-Sud 11, 32 rue

des Carnets, Clamart, France

${ }^{11}$ Immunologie, Hôpital Pitié

Salpétrière, Paris, France

†Deceased. This paper is

dedicated to his memory.

${ }^{*} \mathrm{GC}$ and PR contributed equally

to the study.

\section{Correspondence to}

Xavier Mariette, Service de

Rhumatologie, Hôpital de

Bicêtre, 78 rue du Général

Leclerc, 94275 Le Kremlin

Bicêtre, France; xavier.

mariette@bct.aphp.fr

Received 5 July 2011

Accepted 4 December 2011

Published Online First

17 January 2012

\author{
Xavier Mariette, ${ }^{1}$ Gabriel Baron, ${ }^{2}$ Florence Tubach, ${ }^{3}$ Frédéric Lioté, ${ }^{4}$ Bernard Combe, ${ }^{5}$ \\ Corinne Miceli-Richard, ${ }^{1}$ René-Marc Flipo, ${ }^{6}$ Philippe Goupille, ${ }^{7}$ Matthieu Allez, ${ }^{8}$ \\ Dominique Salmon, ${ }^{9}$ Dominique Emiliet ${ }^{10}{ }^{10}$ Guislaine Carcelain*, ${ }^{*}$ Philippe Ravaud $^{* 2}$
}

\begin{abstract}
Background The recommendations for detecting latent tuberculosis infection (LTBI) before antitumour necrosis factor (anti-TNF) therapy are based on the tuberculin skin test (TST), which lacks both specificity and sensitivity and can lead to unnecessary treatment with antibiotics. A study was undertaken to investigate the effect of replacing TST with interferon $\gamma$ (IFN $\gamma$ ) release assays (IGRA) in screening for LTBI and deciding to begin prophylactic antituberculosis (TB) antibiotics before anti-TNF therapy in immune-mediated inflammatory diseases.
\end{abstract}

Methods In 15 tertiary care hospitals, consecutive patients with rheumatoid arthritis, spondylarthropathies or Crohn's disease were screened for LTBI before anti-TNF therapy with TST, QuantiFERON TB Gold in tube (OTFGold IT) and T-SPOT.TB at the same time. The potential diagnosis of LTBI and the effect on the decision to begin antibiotic prophylaxis were assessed.

Results Among 429 patients, 392 had results for the three tests. The results for TST, T-SPOT.TB and OTF Gold IT were positive for $35.2 \%, 15.1 \%$ and $9.9 \%$ of patients, respectively $(p<0.0001)$. Antibiotics were required for 177 patients (45.2\%) if positive TST results were included in the LTBI definition, 107 patients (27.3\%) if TST results were replaced with results from one of the IGRA tests and 84 patients (21.4\%) if TST results were replaced with OTF-Gold IT results $(p<0.0001)$. The decision on the use of antibiotic prophylaxis was changed for 113 patients (28.8\%, 95\% Cl 24.4\% to 33.6\%) if TST results were replaced with QTF-Gold IT results.

Conclusions Replacing TST with IGRA for determining LTBI allowed the proportion of patients with immunemediated inflammatory diseases needing prophylactic anti-TB antibiotics before beginning anti-TNF agents to be reduced by half.

TrialRegNo: NCT00811343.

\section{INTRODUCTION}

Antitumour necrosis factor (anti-TNF) agents are approved for the treatment of several immunemediated inflammatory diseases including rheumatoid arthritis (RA), spondylarthropathies (SpA), Crohn's disease (CD), psoriasis and juvenile idiopathic arthritis and provide marked clinical benefit. However, one of the most problematic severe adverse effects of these drugs is the possible reactivation of latent tuberculosis (TB) infection (LTBI) in patients previously exposed to TB bacilli. ${ }^{12}$ The presence of quiescent mycobacteria defines LTBI. ${ }^{3}$ Thus, screening for LTBI has been recommended before initiating treatment with TNF blockers. ${ }^{4}$ 5 This screening has resulted in a decrease in the incidence of $\mathrm{TB}$, but $\mathrm{TB}$ still remains a problem in patients receiving anti-TNF therapy with an incidence of $0.1-0.5 / 100$ patient-years in western Europe where TB is not endemic. ${ }^{6-8}$

$\mathrm{TB}$ is more frequent in patients receiving monoclonal antibodies than the soluble receptor, ${ }^{7}$ and remains the most frequent opportunistic infection in these patients receiving anti-TNF therapy. ${ }^{9}$

The recommendations for detecting LTBI differ worldwide, ${ }^{4} 51011$ but are based mainly on the tuberculin skin test (TST) which has numerous drawbacks. The TST requires a return visit for reading the test result and it has poor specificity because previous vaccination with bacillus of Calmette and Guerin (BCG) and environmental mycobacterial exposure can result in false-positive results. ${ }^{10} 1213$ This poor specificity can lead to unnecessary treatment with antibiotics with the possible risk of drug toxicity. ${ }^{14-16}$ However, TST results for patients with immune-mediated inflammatory diseases (IMID) are in general lower because of the disease or immunosuppressive drug use. ${ }^{1718}$ This poor sensitivity can lead to false-negative results, with a subsequent risk of $\mathrm{TB}$ reactivation with anti-TNF therapy. Indeed, two-thirds (30/45) of patients with IMID and TB in the French RATIO registry had a TST result $<5 \mathrm{~mm}$, a negative result. ${ }^{8}$

Identification of genes in the TB genome that are absent in BCG and most environmental mycobacteria offers an opportunity to develop more specific tests to investigate TB infection, particularly LTBI. ${ }^{19}$ Ex vivo interferon $\gamma($ IFN $\gamma)$ release assays (IGRAs) investigating IFN $\gamma$ release by $\mathrm{T}$ cells in the presence of these specific mycobacterial antigens, QuantiFERON TB Gold in tube (OFT-Gold IT) (Cellestis, Carnegie, Australia) and T-SPOT.TB (Oxford Immunotec, Abingdon, UK), seem to be more accurate than the TST for diagnosing active $\mathrm{TB}$, recent primary infection or LTBI in the general population. ${ }^{13}$ 
Table 1 Characteristics of patients

\begin{tabular}{|c|c|c|c|c|c|}
\hline & & $\mathrm{RA}(\mathrm{N}=123)$ & SpA $(N=178)$ & $C D(N=91)$ & Total $(\mathrm{N}=392)$ \\
\hline Age, median (IOR) & & $56(45-53)$ & $42(33-51)$ & $36(27-46)$ & $45(34-56)$ \\
\hline Sex, M/F & & $26 / 97$ & $94 / 83$ & $42 / 47$ & $162 / 227$ \\
\hline Birth in endemic zone of TB & & $18(14.6)$ & $24(13.6)$ & $16(17.6)$ & $58(14.8)$ \\
\hline Corticosteroids or immunosuppressants & & $114(92.7)$ & $64(36.0)$ & $56(61.5)$ & $234(59.7)$ \\
\hline Corticosteroids & & $86(69.9)$ & $24(13.5)$ & $30(33.0)$ & $140(35.7)$ \\
\hline Immunosuppressants & & $103(83.7)$ & $55(30.9)$ & $40(44.0)$ & $198(50.5)$ \\
\hline \multirow[t]{3}{*}{ BCG vaccination } & No & $9(7.3)$ & $5(2.8)$ & $2(2.2)$ & $16(4.1)$ \\
\hline & Yes & $65(52.8)$ & $132(74.6)$ & $60(65.9)$ & $257(65.7)$ \\
\hline & Unknown & $49(39.8)$ & $40(22.6)$ & $29(31.9)$ & $118(30.2)$ \\
\hline LTBI defined by questioning & & $23(18.7)$ & $23(12.9)$ & $7(7.7)$ & $53(13.5)$ \\
\hline LTBI defined by x-ray & & $3(2.5)$ & $3(1.7)$ & $0(0.0)$ & $6(1.6)$ \\
\hline LTBI defined by questioning or x-ray & & $24(19.5)$ & $26(14.6)$ & $7(7.7)$ & $57(14.5)$ \\
\hline
\end{tabular}

Data are number (\%) unless indicated otherwise.

BCG, bacillus of Calmette and Guerin; CD, Crohn's disease; LBTI, latent tuberculosis infection; RA, rheumatoid arthritis; SpA, spondylarthropathies, TB, tuberculosis.
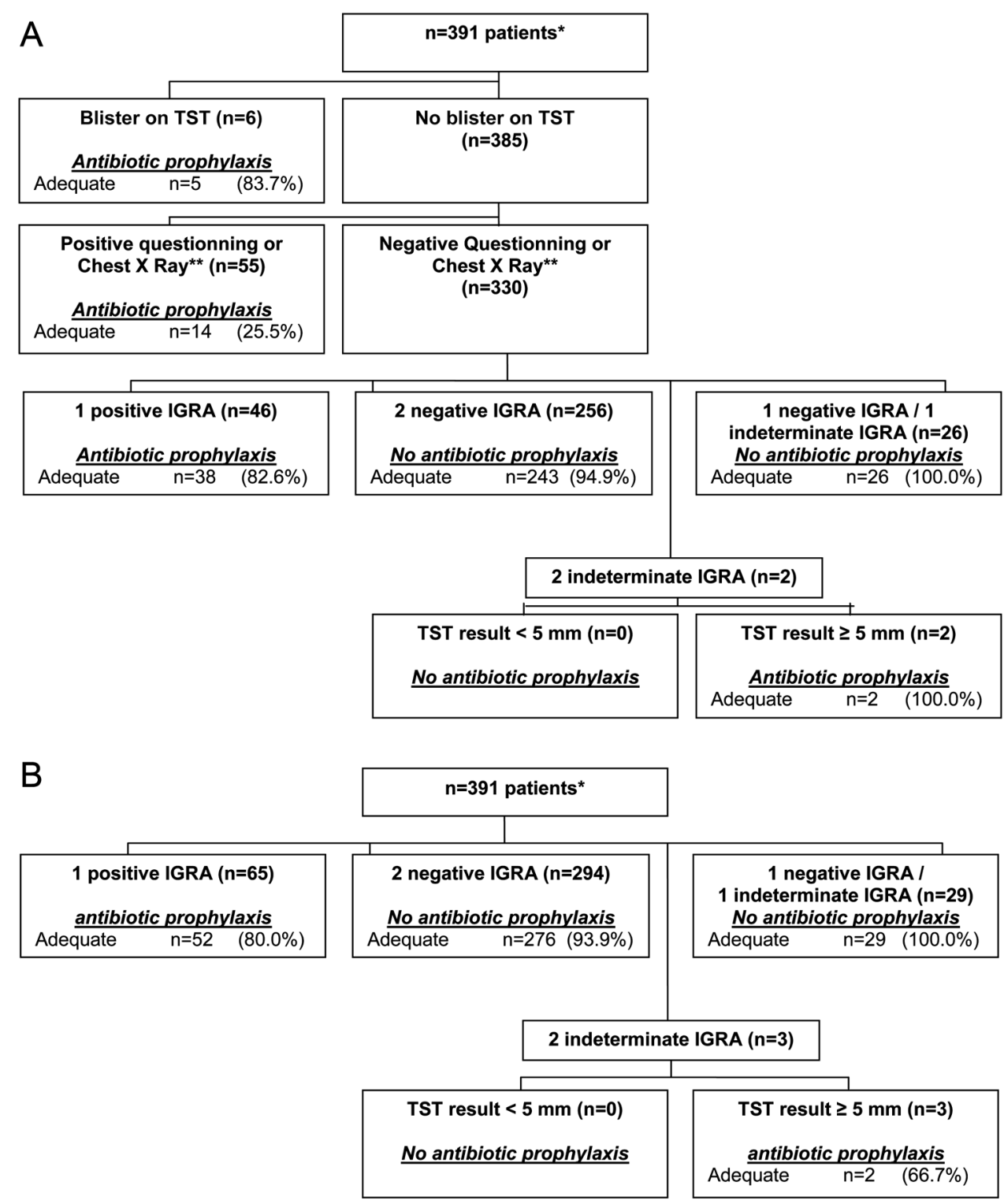

\footnotetext{
*1 missing data for Anti-TB antibiotic prophylaxis

** questioning reveals previous TB without adequate treatment or primary TB infection without adequate treatment or previous confirmed close contact with a patient having pulmonary TB or chest X-ray shows residual nodular tuberculous lesions $>1 \mathrm{~cm}$ in the absence of previous adequate TB treatment
}

Figure 1 Flow chart of patients effectively receiving antibiotic prophylaxis depending on (A) what was planned in the protocol and (B) what was expected from the results of interferon $\gamma$ (IFN $\gamma$ ) release assays (IGRA). TST, tuberculin skin test. 
Table 2 Comparison of interferon $\gamma($ IFN $\gamma$ ) release assay and TST, with a threshold for TST of $5 \mathrm{~mm}$ or $10 \mathrm{~mm}$

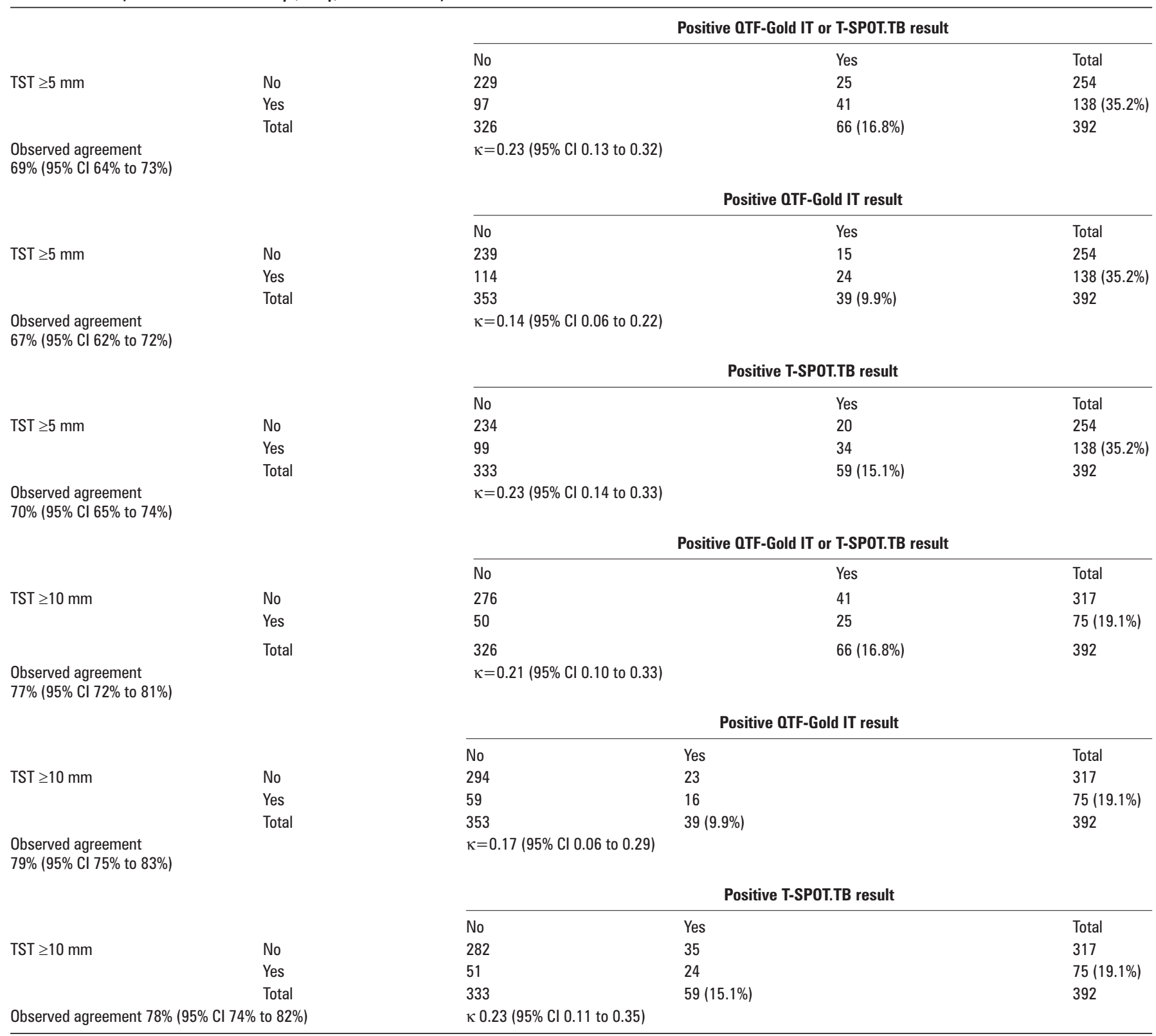

QTF-Gold IT, QuantiFERON TB Gold in tube; TST, tuberculin skin test.

In this large prospective study we aimed to investigate the potential influence in clinical practice of replacing the TST with the OFT-Gold IT and/or T-SPOT.TB for diagnosis of LTBI in deciding on prophylactic anti-TB antibiotic therapy before antiTNF therapy in patients with IMID.

\section{METHODS}

\section{Study design}

A prospective pragmatic multicentre study was conducted to assess the effect of replacing the TST with IGRAs on the diagnosis of LTBI and on the physician's decision on treatment for patients with IMID (ie, the percentage of patients for whom the decision for antibiotic prophylaxis changed).

\section{Patients and follow-up}

Consecutive patients with RA, SpA or CD with an indication for initial biological treatment with anti-TNF agents in 14 rheumatology departments and five gastroenterology departments from 15 tertiary care hospitals were included in the study. In accordance with the French recommendations for screening for LTBI, ${ }^{20}$ all patients underwent a clinical examination focused on questioning about previous contact with TB and a chest x-ray.

Patients were followed for 1 year. Antibiotic treatment, side effects of antibiotics and eventual occurrence of TB were recorded. All patients gave their informed consent to participate in the study.

\section{Tests studied}

Before anti-TNF therapy was started, patients underwent the TST and a blood sample was taken for OFT-Gold IT and T-SPOT.TB within 3 days.

The TST involved intradermal injection of five tuberculin units corresponding to $0.1 \mathrm{ml}$ of purified protein derivative (Tubertest, Sanofi Pasteur, France) according to the intradermal Mantoux method. The main diameter of skin induration was recorded in millimeters by an experienced clinician $72 \mathrm{~h}$ after 
the inoculation and the result was considered positive if skin induration was $\geq 5 \mathrm{~mm}$.

IGRAs were performed following training of staff in 15 hospital laboratories and interpreted by local immunologists according to the manufacturers' instructions who were blind to the TST results. The cut-off value for a positive T-SPOT.TB result was $>7$ spots. Indeterminate results were defined as an IFN $\gamma$ level $\geq 2.0 \mathrm{IU} / \mathrm{ml}$ in the negative control well or $<0.5 \mathrm{IU} / \mathrm{ml}$ in the positive control well for QTF-Gold IT and $>10$ spot-forming cells (SFCs) in the negative control well or $<20$ SFCs in the positive control well for T-SPOT.TB.

\section{Assessment of diagnosis and influence on physician's therapeutic decision}

According to French recommendations, ${ }^{20}$ patients with LTBI require prophylactic antibiotic treatment under at least one of the following three conditions: (1) questioning reveals previous TB without adequate treatment, primary TB infection without adequate treatment, or previous confirmed close contact with a patient having pulmonary TB; (2) the chest x-ray shows residual nodular tuberculous lesions $\geq 1 \mathrm{~cm}$ in the absence of previous adequate TB treatment; or (3) wheals $\geq 5 \mathrm{~mm}$ in diameter on TST. In this study, for a diagnosis of LTBI followed by a decision to give antibiotic prophylaxis treatment, we used conditions 1 and 2 but replaced TST positivity with IGRA positivity (at least one positive result for QTF-Gold IT or T-SPOT. TB), except if TST yielded a blister on skin. In the case of one indeterminate blood test result, the other test result was used. If both blood test results were indeterminate, the TST result was used.

\section{Sample size}

The main objective of this study was to estimate the proportion of patients in whom the decision to administer antibiotic prophylaxis would change. We hypothesised that this change might occur in $20 \%$ of patients. To estimate this proportion with a two-sided $95 \%$ exact CI (based on a binomial distribution) and a precision of $4 \%, 385$ patients were required.

\section{Statistical analysis}

The influence on the physician's decision was assessed by the McNemar test, which was used to compare paired proportions of patients needing antibiotics according to the definition of LTBI (defined by tests (TST, IGRA) and/or questioning or chest $\mathrm{x}$-ray).

The influence on the decision whether or not to administer antibiotic prophylaxis was also computed by determining the proportion of patients with a change in decision for antibiotic prophylaxis (with an exact $95 \%$ binomial CI for proportions).

Table 3 Comparison of interferon $\gamma$ (IFN $\gamma$ ) release assay and TST in the 57 patients with LTBI defined by questioning or $x$-ray

\begin{tabular}{|c|c|c|c|c|c|c|}
\hline Test & $\mathbf{N}$ & Sensitivity & $95 \% \mathrm{Cl}$ & $\mathbf{N}$ & Specificity* & $95 \%$ Cl \\
\hline TST & 57 & 0.32 & $\begin{array}{l}0.20 \text { to } \\
0.45\end{array}$ & 335 & 0.54 & $\begin{array}{l}0.69 \text { to } \\
0.70\end{array}$ \\
\hline QTF-Gold IT & 57 & 0.21 & $\begin{array}{l}0.11 \text { to } \\
0.34\end{array}$ & 335 & 0.92 & 0.89 to 0.95 \\
\hline T-SPOT.TB & 57 & 0.25 & $\begin{array}{l}0.14 \text { to } \\
0.38\end{array}$ & 335 & 0.87 & 0.82 to 0.90 \\
\hline $\begin{array}{l}\text { OTF-Gold IT or } \\
\text { T-SPOT.TB }\end{array}$ & 57 & 0.28 & $\begin{array}{l}0.17 \text { to } \\
0.42\end{array}$ & 335 & 0.85 & 0.81 to 0.89 \\
\hline
\end{tabular}

*Indeterminate and negative results were pooled.

LTBI, latent tuberculosis infection; QTF-Gold IT, QuantiFERON TB Gold in tube; TST, tuberculin skin test
To identify factors associated with positive TST or IGRA results, we performed bivariate analysis ( $\chi^{2}$ tests). The following factors were tested: age, pathology, birth in an endemic TB zone, treatment with corticosteroids or immunosuppressive drugs or corticosteroids alone, BCG vaccination, LTBI defined by questioning alone or chest $\mathrm{x}$-ray. Multiple logistic regression analysis was then performed and variables with $p \leq 0.2$ on bivariate analysis were included in the model. Variables retained in the final multivariate model were selected by a backward procedure. ORs and $95 \%$ CI were computed. The same methodology was used to find factors associated with an indeterminate IGRA result (OFT-Gold IT or T-SPOT.TB).

All analyses were performed using SAS 9.1 (SAS Institute, Cary, North California, USA) and a $\mathrm{p}<0.05$ was considered statistically significant.

\section{RESULTS}

\section{Patient characteristics}

We included 429 patients (table 1) from 15 hospitals (14 rheumatology centres and five gastroenterology centres) who had not previously received biological agents. IGRA findings were analysed in the local immunology laboratories. Results for the three tests (TST and both IGRAs) were available for 392 patients $(91.4 \%)$.

Overall, 57 patients (14.5\%) had a diagnosis of LTBI made independently of the TST or IGRA results (based on previous TB contact without adequate treatment on questioning or abnormal chest x-ray results).

\section{Outcome and compliance with the planned intervention}

Twenty-seven of the 392 patients (6.9\%) were lost to follow-up at 1 year. None of the 365 patients who completed the 1-year follow-up had TB.

The decision whether or not to administer anti-TB antibiotic prophylaxis according to the protocol or only the IGRA test results was in compliance with the planned intervention in $83.4 \%$ and $91.8 \%$ of the patients, respectively (figure 1). All but four patients $(94 \%)$ received the combination of isoniazid and rifampicin for 3 months.

\section{Comparison between TST and IGRA results}

The direct comparison of TB screening tests is shown in table 2. TST results were positive for $35.2 \%$ of patients (indicating LTBI) whereas the results for either IGRA were positive for $16.8 \%$ $(p<0.0001)$. The number of patients with discordant results was high: 122 (31.1\%, 95\% CI $26.6 \%$ to $36.0 \%$ ) for all discordant results, $97(80 \%)$ with positive TST and negative IGRA results and $25(20 \%)$ with negative TST and positive IGRA results. If we considered only OFT-Gold IT, the difference from TST was greater: $9.9 \%$ with positive OFT-Gold IT results versus $35.2 \%$ with positive TST results $(\mathrm{p}<0.0001$; table 2$)$.

The percentage of positive TST results with a threshold of TST of $10 \mathrm{~mm}$ and positive IGRA results was similar $(19.1 \%$ vs $16.8 \%, p=0.35$ ) but the number of patients with discordant results remained high (91 (23.2\%), $95 \%$ CI $19.1 \%$ to $27.7 \%$, table 2 ).

Patients with each IMID showed differences in positivity between TST and IGRA results (see table 1 in online supplement).

The frequency of positive IGRA results was the same whether or not patients received corticosteroids or immunosuppressants (any disease-modifying antirheumatic drug or azathioprine) (18.6\% and $17.1 \%$, respectively). Conversely, TST results were less frequently positive for patients receiving corticosteroids 
or immunosuppressants than for those receiving no treatment (31.2\% vs $41.1 \%, \mathrm{p}=0.04)$.

With regard to the 57 patients considered to have LTBI on questioning or x-ray independently from the tests, the sensitivity of TST and IGRA was equally low but the specificity of IGRA was higher (table 3).

The agreement rate between the two IGRA assays was 91\% (95\% CI $88 \%$ to $94 \%$ ) with a $\kappa$ coefficient of 0.61 (95\% CI 0.49 to 0.73 ) (see table 2 in online supplement).

\section{Diagnosis and influence on physician's decision to administer antibiotic prophylaxis in patients with LTBI defined by tests and questioning or chest $\mathrm{x}$-ray}

When a positive response to questioning or a positive chest $\mathrm{x}$-ray was added to positive TST results with a threshold of 5 $\mathrm{mm}, 177$ patients $(45.2 \%)$ had LTBI. TST results were replaced by one or the other positive IGRA results and 107 patients $(27.3 \%)$ were then considered to have LTBI ( $45.2 \%$ vs $27.3 \%$, $\mathrm{p}<0.0001$ ) and received antibiotic prophylaxis (table 4$)$. The number of patients in whom there was a change in the decision on antibiotic prophylaxis was 106/392 (ie, the influence on the physician's therapeutic decision was $27.0 \%$, 95\% CI $22.7 \%$ to $31.7 \%$ ). Among these 106 patients, 88 (83\%) had positive TST and negative IGRA results and $18(17 \%)$ had negative TST and positive IGRA results.

If TST results were replaced with only OFT-Gold IT results, 84 patients $(21.4 \%)$ were considered to have LTBI $(45.2 \%$ vs $21.4 \%, p<0.0001)$. The number of patients with a change in the decision on antibiotic prophylaxis was 113 (ie, the influence on the physician's therapeutic decision was $28.8 \%$, 95\% CI $24.4 \%$ to $33.6 \%$ ).

\section{Factors associated with positive TST or IGRA results}

The factors associated with positive OFT-Gold IT, T-SPOT. TB and TST results on bivariate and multivariate analysis are shown in table 5. Importantly, OFT-Gold IT positivity was associated with LTBI defined by only questioning or x-ray, birth in an endemic TB zone, absence of or unknown BCG vaccination and age. Risk factors associated with T-SPOT.TB positivity and with TST positivity were having RA or SpA versus CD, birth in an endemic TB zone and age.

Even though previous BCG vaccination was not found to be a risk factor for TST positivity, the 97 patients with positive TST and negative IGRA results had received a previous BCG vaccination more frequently than the other 295 patients $(76.3 \%$ vs $62.0 \%, p=0.02)$. Moreover, the frequency of TST positivity was the same in patients with or without BCG vaccination (37.7\% and $37.5 \%$, respectively), whereas it was very different for IGRA (15.6\% and $43.8 \%$, respectively) (see table 3 in online supplement). Interestingly, in the small group of patients without BCG vaccination ( $n=16$ ), the observed proportion of agreement between IGRA and TST was good $(81.3 \%, 95 \%$ CI $54.4 \%$ to $81.2 \%$ ), much better than in patients with previous BCG vaccination $(\mathrm{n}=257)(64.6 \%, 95 \%$ CI $58.4 \%$ to $70.4 \%)$.

\section{Factors associated with indeterminate test results}

The percentage of indeterminate test results was lower with OFT-Gold IT than with T-SPOT.TB $(2.8 \%$ vs $6.4 \%, \mathrm{p}=0.01)$. Among the 11 indeterminate test results with OFT-Gold IT, 10 were due to a low IFN $\gamma$ response in the positive control mitogen tube and eight had a negative T-SPOT.TB result. Among the 25 indeterminate test results with T-SPOT.TB, 16 were due to a high background IFN $\gamma$ level in the negative control nil tube and most were negative OTF-Gold IT results (14/16). Only four indeterminate test results with T-SPOT.TB were due to a low mitogen response and there were five difficulties in interpretation.

No factor was associated with an indeterminate IGRA result (indeterminate OFT-Gold IT or T-SPOT TB result) and, in particular, neither steroid nor immunosuppressant use.

\section{DISCUSSION}

In this study of 392 patients with IMID in whom two IGRAs and TST were evaluated before beginning anti-TNF therapy, we have shown that replacing TST with IGRA in screening for LTBI allowed the proportion of patients requiring antibiotic prophylaxis to be reduced by half. Taking into account the number of diseases with an indication for anti-TNF therapy and the possible inconvenience of antibiotic prophylaxis (cost, possible side effects, delay in anti-TNF treatment), the conclusions of this study have important practical consequences.

IGRAs have been validated for detecting LTBI in unselected and HIV-positive populations ${ }^{13}$ but, despite its widely recognised limitations, the TST remains the most generally used method for screening for LTBI before anti-TNF therapy. The difficulty in evaluating IGRAs for this indication is the lack of a gold standard for defining LTBI and the fact that the outcome we aim to prevent by screening for LTBI represents a significant concern but occurs too infrequently to design studies for comparison of the

Table 4 Comparison of patients with and without LTBI including either IGRA or TST (threshold $5 \mathrm{~mm}$ ) in the algorithm

\begin{tabular}{|c|c|c|c|c|}
\hline & & \multicolumn{3}{|c|}{ LTBI defined by questioning or $x$-ray or a positive result with one of the IGRAs } \\
\hline \multirow{4}{*}{$\begin{array}{l}\text { LTBI defined by questioning or } x \text {-ray or } \\
\text { TST result } \geq 5 \mathrm{~mm}\end{array}$} & & No LTBI & LTBI & Total \\
\hline & No LTBI & 197 & 18 & 215 \\
\hline & LTBI & 88 & 89 & $177(45.2 \%)$ \\
\hline & Total & 285 & $107(27.3 \%)$ & 392 \\
\hline \multicolumn{5}{|c|}{ LTBI defined by questioning or $\mathrm{x}$-ray or positive QTF-Gold IT result } \\
\hline \multirow{4}{*}{$\begin{array}{l}\text { LTBI defined by questioning or } x \text {-ray or } \\
\text { TST result } \geq 5 \mathrm{~mm}\end{array}$} & & No LTBI & LTBI & Total \\
\hline & No LTBI & 205 & 10 & 215 \\
\hline & LTBI & 103 & 74 & 177 (45.2\%) \\
\hline & Total & 308 & $84(21.4 \%)$ & 392 \\
\hline \multirow{5}{*}{$\begin{array}{l}\text { LTBI defined by questioning or } x \text {-ray or } \\
\text { TST result } \geq 5 \mathrm{~mm}\end{array}$} & & \multicolumn{3}{|c|}{ LTBI defined by questioning or x-ray or positive T-SPOT.TB result } \\
\hline & & No LTBI & LTBI & Total \\
\hline & No LTBI & 200 & 15 & 215 \\
\hline & LTBI & 90 & 87 & $177(45.2 \%)$ \\
\hline & Total & 290 & $102(26.0 \%)$ & 392 \\
\hline
\end{tabular}


different screening methods. The ideal study design would be to compare the incidence of reactivation of TB in patients receiving prophylactic antibiotics according to standard recommendations including TST and those including IGRA. Taking into account the incidence of reactivation of $\mathrm{TB}$ in patients screened using standard recommendations (about $1 / 1000$ patients starting anti-TNF therapy in the $\mathrm{BSRBR}^{7}$ and the RATIO registry), ${ }^{8}$ this study would need 5000-10 000 patients in each group, which is unrealistic. Thus, the only way to assess the usefulness of these new tests is to compare both tests and TST with classic risk factors of LTBI in a sufficiently large sample of homogeneous patients with IMID with no previous biotherapy and to include the IGRA in the decision on whether or not to prescribe TB antibiotic prophylaxis. To date, some reports of the use of IGRA in IMID have been published, but most have included few patients, are heterogeneous concerning the time of the tests (before or during anti-TNF treatment) or did not perform the two IGRAs and the TST at the same time. ${ }^{21-27}$ However, some of these studies gave indications concordant with our results, with a decrease in patients requiring antibiotic prophylaxis and a better association with classic risk factors for LTBI with IGRA than with the TST. ${ }^{23-26}$

There is no gold standard for defining LTBI and thus we cannot calculate the sensitivity and specificity of the tests. We can approach these data by focusing on the 57 patients considered to have LTBI on questioning or x-ray independently from the tests. However, we have to keep in mind that the diagnosis of putative LTBI in these patients is based mainly on questioning and not on objective features. In these patients, the sensitivity of TST and IGRA was equally low but the specificity of IGRA was higher (table 3 ).

The better specificity of IGRAs was confirmed by the fact that, with the classic method of screening for LTBI (ie, including TST), $45.2 \%$ of the patients with IMID should receive antibiotic prophylaxis. If TST is replaced with one of the IGRAs, this percentage falls to $27.3 \%$, and to $21.4 \%$ if only QTF-Gold IT results are considered. The percentage of patients with at least one positive IGRA result is twice as low as that of patients with

Table 5 Factors associated with positive OTF-Gold IT, positive T-Spot.TB and positive TST results in bivariate and multivariate analysis

\begin{tabular}{|c|c|c|c|c|c|c|}
\hline & \multicolumn{3}{|l|}{ Bivariate analysis } & \multicolumn{3}{|c|}{ Multivariate analysis } \\
\hline & $\begin{array}{l}\text { Positive 0TF-Gold IT } \\
\text { result }(\mathrm{n}=39)\end{array}$ & $\begin{array}{l}\text { Negative or indeterminate } \\
\text { QTF-Gold IT result }(n=353)\end{array}$ & p Value & $\mathbf{O R}$ & $95 \% \mathrm{CI}$ & p Value \\
\hline Age, median (IQR)/(OR per 10 years) & $53(43-64)$ & $44(33-44)$ & 0.0006 & 1.42 & 1.09 to 1.85 & 0.0081 \\
\hline RA and $\mathrm{SpA}$ (vs CD) & $32(82.1)$ & $269(76.2)$ & 0.4118 & & & \\
\hline Birth in endemic TB zone & $12(30.8)$ & $46(13.1)$ & 0.0032 & 2.97 & 1.33 to 6.66 & 0.0081 \\
\hline Corticosteroids or immunosuppressors & $26(66.7)$ & $2(58.9)$ & 0.3495 & & & \\
\hline Corticosteroids alone & $19(48.7)$ & $121(34.3)$ & 0.0741 & & & \\
\hline BCG vaccination & & & 0.0005 & & & 0.0357 \\
\hline No & $6(15.4)$ & $10(2.8)$ & & 1 & & \\
\hline Yes & $20(51.3)$ & $237(67.1)$ & & 0.23 & 0.07 to 0.73 & \\
\hline Unknown & $13(33.3)$ & $106(30.0)$ & & 0.23 & 0.07 to 0.77 & \\
\hline \multirow{2}{*}{$\begin{array}{l}\text { LTBI defined by questioning alone* } \\
\text { or chest } x \text {-ray } \dagger\end{array}$} & $12(30.8)$ & $45(12.7)$ & 0.0024 & 2.30 & 1.04 to 5.11 & 0.0407 \\
\hline & $\begin{array}{l}\text { Positive T-SPOT.TB } \\
\text { result }(n=59)\end{array}$ & $\begin{array}{l}\text { Negative or indeterminate } \\
\text { T-SPOT.TB result }(n=333)\end{array}$ & $\mathrm{p}$ Value & $\mathrm{OR}$ & $95 \% \mathrm{Cl}$ & $\mathrm{p}$ Value \\
\hline Age, median (IOR)/(OR per 10 years) & $51(39-60)$ & $44(33-54)$ & 0.0025 & 1.27 & 1.03 to 1.57 & 0.0232 \\
\hline RA and $\mathrm{SpA}$ (vs CD) & $53(89.8)$ & $248(74.5)$ & 0.0100 & 2.69 & 1.08 to 6.71 & 0.0342 \\
\hline Birth in endemic TB zone & $17(28.8)$ & $41(12.3)$ & 0.0010 & 3.11 & 1.59 to 6.10 & 0.0009 \\
\hline Corticosteroids or immunosuppressors & $36(61.0)$ & $198(59.5)$ & 0.8221 & & & \\
\hline Corticosteroids alone & $23(39.0)$ & $117(35.1)$ & 0.5697 & & & \\
\hline BCG vaccination & & & 0.1805 & & & \\
\hline No & $5(8.5)$ & $11(3.3)$ & & & & \\
\hline Yes & $37(62.7)$ & $220(66.1)$ & & & & \\
\hline Unknown & $17(28.8)$ & $102(30.6)$ & & & & \\
\hline \multirow{2}{*}{$\begin{array}{l}\text { LTBI defined by questioning alone* } \\
\text { or chest x-ray } \dagger\end{array}$} & $14(23.7)$ & $43(12.9)$ & 0.0298 & & & \\
\hline & $\begin{array}{l}\text { Positive TST } \\
\text { result }(n=138)\end{array}$ & $\begin{array}{l}\text { Negative TST } \\
\text { result }(n=254)\end{array}$ & $\mathrm{p}$ Value & $\mathrm{OR}$ & $95 \% \mathrm{Cl}$ & $\mathrm{p}$ Value \\
\hline Age, median (IOR)/(OR per 10 years) & $44(36-51)$ & $45(33-58)$ & 0.1619 & 0.80 & 0.68 to 0.94 & 0.0059 \\
\hline RA and $\mathrm{SpA}$ (vs CD) & $120(87.0)$ & $181(46.2)$ & 0.0004 & 3.76 & 2.04 to 6.91 & $<0.0001$ \\
\hline Birth in endemic TB zone & $28(20.3)$ & $30(11.9)$ & 0.0250 & 2.15 & 1.19 to 3.88 & 0.0108 \\
\hline Corticosteroids or immunosuppressors & $73(52.9)$ & $161(63.4)$ & 0.0432 & & & \\
\hline Corticosteroids alone & $41(29.7)$ & $99(39.0)$ & 0.0674 & & & \\
\hline BCG vaccination & & & 0.2846 & & & \\
\hline No & $6(4.3)$ & $10(3.9)$ & & & & \\
\hline Yes & $97(70.3)$ & $160(63.0)$ & & & & \\
\hline Unknown & $35(25.4)$ & $84(33.1)$ & & & & \\
\hline LTBI defined by questioning alone* or chest x-ray $\dagger$ & $18(13.0)$ & $39(15.4)$ & 0.5353 & & & \\
\hline
\end{tabular}

Data are number (\%) unless otherwise specified.

*TB primary infection, close contact with TB patient, TB with no treatment, anti-TB treatment initiated before 1970 or less than 2 months with the combination rifampicinpyrazinamide treatment.

†Nodules $>1 \mathrm{~cm}$.

BCG, bacillus of Calmette and Guerin; LTBI, latent tuberculosis infection; QTF-Gold IT, QuantiFERON TB Gold in tube; RA, rheumatoid arthritis; SpA, spondylarthropathies; TB, tuberculosis; TST, tuberculin skin test. 
positive TST results $(16.8 \%$ vs $35.2 \%)$. This $16.8 \%$ rate of history of contact with TB bacillus is high, but is in accordance with previous studies in this type of population of the same age..$^{23-25}$ The sensitivity of IGRA is also probably better than that of TST because, in $20 \%$ of the discordant results, the TST result was negative whereas the IGRA results were positive. This finding is probably due to the fact that the frequency of positive IGRA results, but not of TST results, was the same whether patients received corticosteroids or immunosuppressants.

Finally, an important argument in favour of the validity of the IGRA is the association with classic risk factors for LTBI. Both IGRA and TST results are associated with birth in endemic areas of TB, whereas OFT-Gold IT results alone are associated with LTBI defined only by questioning or by x-ray and with an absence of BCG vaccination. Our data therefore suggest that BCG vaccination can protect from positive IGRA and, even though not found as an independent risk factor of TST positivity, can favour a non-specific positive TST result. Interestingly, among the six patients with blister on TST, five of six had a positive IGRA result and the one patient with a negative IGRA test had previous BCG vaccination.

If we consider the two IGRAs independently as in the study by Kleinert et al, ${ }^{26}$ OFT-Gold IT but not T-SPOT.TB results were associated with classic risk factors for LTBI, probably because of the better specificity of OFT-Gold IT. ${ }^{28}$ Supporting this assertion, among the 27 patients with positive T-SPOT.TB and negative OFT-Gold IT TB results, the frequency of LTBI defined by questioning alone or $\mathrm{x}$-ray was the same as in the whole population $(14.8 \%$ vs $14.5 \%)$. However, as found in recent meta-analyses ${ }^{29}$ 30 on the effect of these tests in diagnosing active TB, T-SPOT. TB had better sensitivity than OFT-Gold IT in our study $(15.1 \%$ vs $9.9 \%$ ). As no gold standard exists, it is not possible to say if the better sensitivity of T-SPOT.TB corresponds to false positives. In fact, most of the discordant results (positive T-SPOT.TB and negative OFT-Gold IT) were in patients with SpA, with less immunosuppressant treatment suggesting that the number of positive spots for defining the threshold of positivity of T-SPOT. TB could be too low in patients without immunosuppressant treatment.

Finally, indeterminate results were less frequent with QTFGold IT than with T-SPOT.TB $(2.8 \%$ vs $6.4 \%)$, which is similar to that reported for the general adult population ${ }^{28}$ and also in a small series of patients with IMID. ${ }^{31}$ Interestingly, the high number of indeterminate results for T-SPOT.TB was mainly due to a high background IFN $\gamma$ level in the negative control well for patients with negative OFT-Gold IT results.

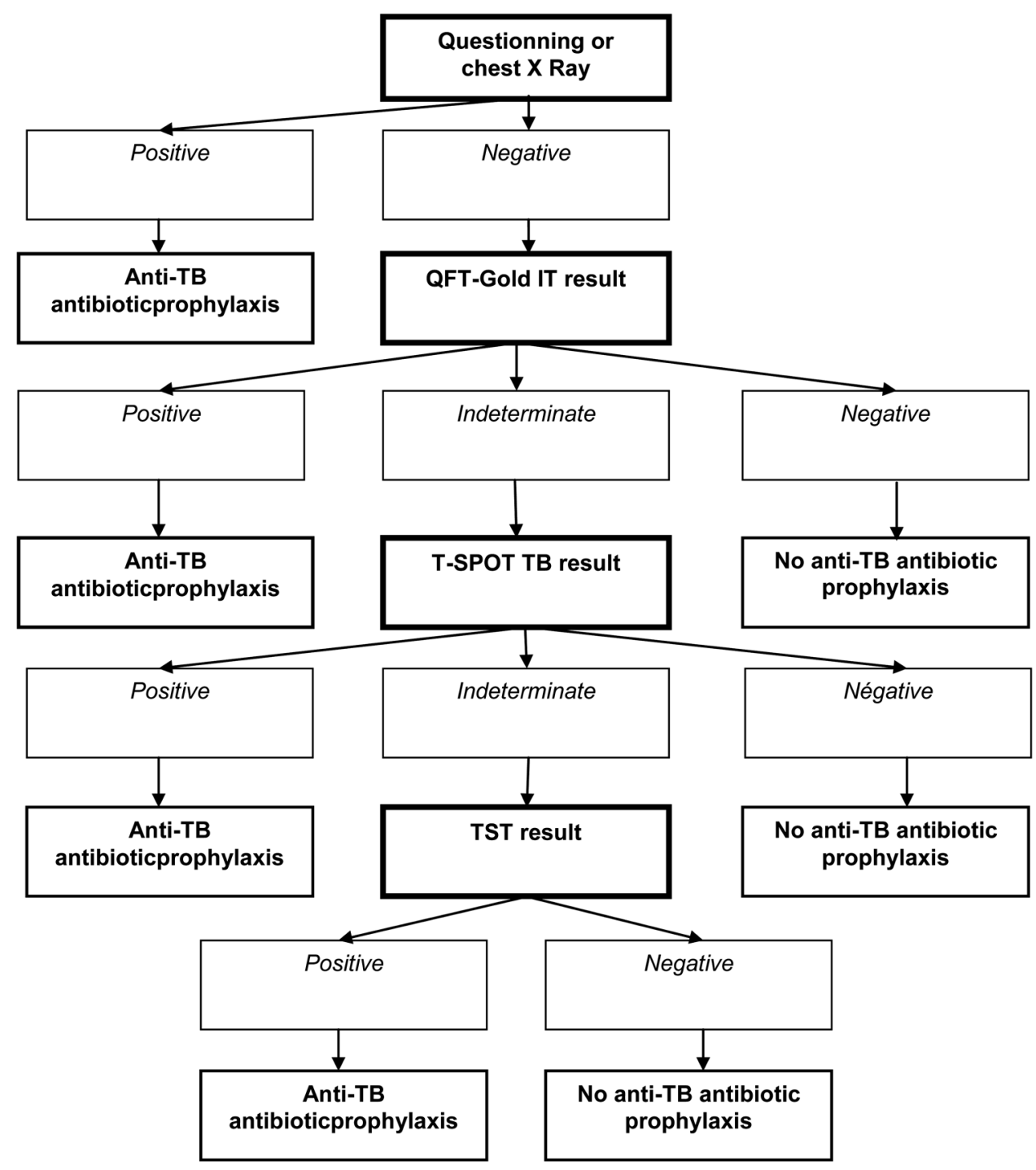

Figure 2 Proposed algorithm for screening for latent tuberculosis (TB) infection before anti-tumour necrosis factor treatment. TST, tuberculin skin test. 
Together these results support the fact that using IGRAs (instead of TST) results in fewer patients with IMIDs with an indication for anti-TNF therapy being diagnosed and treated for LTBI. Our data support recent expert recommendations ${ }^{32}$ suggesting, without evidence, that IGRAs are superior to the TST in identifying individuals with a prior history of BCG vaccination at risk of developing TB with anti-TNF therapy. $x$-Rays do not appear to be informative for this purpose, but baseline reference x-rays are useful before starting anti-TNF therapy and we therefore propose that they should be left in the algorithm. The question of performing one or both IGRAs remains debatable. For economic, practical (compared with T-SPOT.TB, OFT-Gold IT does not require Ficoll separation and thus can be fully automatic) and scientific reasons stated previously, we propose the following algorithm: questioning, chest x-ray and OFT-Gold IT testing. T-SPOT.TB should be performed in cases with indeterminate OFT-Gold IT results and TST in cases with indeterminate results for both IGRAs (figure 2). With this algorithm, the proportion of patients requiring anti-TB antibiotic prophylaxis would decrease from $45.2 \%$ to $21.4 \%$. This strategy needs to be evaluated from a medico-economic point of view and validated in other cohorts.

Contributors XM, GB, GC and PR had full access to all of the data in the study and take responsibility for the integrity of the data and the accuracy of the data analysis.

Acknowledgements The authors thank S Makhlouf and N Nicolas (Unité de Recherche Clinique, Hôpital Bichat-Claude Bernard) for their help in collecting cases. They thank all the investigators of the ETAT Study Group: 15 immunology laboratories: Dr S Benzaken (Nice), Dr F Bienvenu (Lyon), Dr G Carcelin (Paris), Dr A Chevallier (Angers), Dr J M Gomberg (Poitiers), Dr B Heym (Paris), Dr M Labalette (Lille), Dr C Lambert (Saint-Étienne), Dr S Martin (Paris), Dr D Monnier (Rennes), Dr C Rabian (Paris), Dr Y Taoufik (Paris), Dr G Thibault (Tours), Dr J P Vendrell (Montpellier). 14 rheumatology departments: Pr M Audran (Angers), Pr T Bardin (Paris), Pr P Orcel (Paris), Pr P Bourgeois (Paris), Pr B Combe (Montpellier), Pr F Debiais (Poitiers), Pr L Euller Ziegler (Nice), Pr R M Flipo (Lille), Pr J M Le Parc (Paris), Pr X Mariette (Paris), Pr 0 Meyer (Paris), Pr A Perdriger (Rennes), Pr T Thomas (Saint-Etienne); five gastroenterology departments: Pr M Allez (Paris), Pr J F Colombel (Lille), Pr X Hebuterne (Nice), Dr A L Pelletier (Paris), Dr L Picon (Tours).

Funding This study was funded by the French Ministry of Health thanks to a STIC (Stratégies thérapeutiques Innovantes Couteuses) grant. The sponsor was the Département de la Recherche Clinique et du Développement of the Assistance Publique - Hôpitaux de Paris (STIC0717, P070310).

Ethics approval The study was authorised by the ethics committee of Paris lle de France (CPP Ile de France II, № 2008-07-04).

Competing interests None.

Provenance and peer review Not commissioned; externally peer reviewed.

\section{REFERENCES}

1. Keane J, Gershon S, Wise RP, et al. Tuberculosis associated with infliximab, a tumor necrosis factor alpha-neutralizing agent. N Eng/ J Med 2001;345:1098-104.

2. Gómez-Reino JJ, Carmona L, Valverde VR, et al. Treatment of rheumatoid arthritis with tumor necrosis factor inhibitors may predispose to significant increase in tuberculosis risk: a multicenter active-surveillance report. Arthritis Rheum 2003;48:2122-7.

3. Horsburgh CR Jr. Priorities for the treatment of latent tuberculosis infection in the United States. N Eng/ J Med 2004;350:2060-7.

4. Hamilton CD. Tuberculosis in the cytokine era: what rheumatologists need to know. Arthritis Rheum 2003;48:2085-91

5. Furst DE, Cush J, Kaufmann S, et al. Preliminary guidelines for diagnosing and treating tuberculosis in patients with rheumatoid arthritis in immunosuppressive trials or being treated with biological agents. Ann Rheum Dis 2002;61(Suppl 2):ii62-3

6. Burmester GR, Mariette X, Montecucco C, et al. Adalimumab alone and in combination with disease-modifying antirheumatic drugs for the treatment of rheumatoid arthritis in clinical practice: the Research in Active Rheumatoid Arthritis (ReAct) trial. Ann Rheum Dis 2007;66:732-9.

7. Dixon WG, Hyrich KL, Watson KD, et al. Drug-specific risk of tuberculosis in patients with rheumatoid arthritis treated with anti-TNF therapy: results from the
British Society for Rheumatology Biologics Register (BSRBR). Ann Rheum Dis 2010;69:522-8

8. Tubach F, Salmon D, Ravaud P, et al. Risk of tuberculosis is higher with anti-tumor necrosis factor monoclonal antibody therapy than with soluble tumor necrosis factor receptor therapy: The three-year prospective French Research Axed on Tolerance of Biotherapies registry. Arthritis Rheum 2009;60:1884-94

9. Salmon-Ceron D, Tubach F, Lortholary 0, et al. Drug-specific risk of non-tuberculosis opportunistic infections in patients receiving anti-TNF therapy reported to the 3-year prospective French RATIO registry. Ann Rheum Dis 2011;70:616-23.

10. Keane J. TNF-blocking agents and tuberculosis: new drugs illuminate an old topic. Rheumatology (Oxford) 2005;44:714-20.

11. Jasmer RM, Daley CL. Rifampin and pyrazinamide for treatment of latent tuberculosis infection: is it safe? Am J Respir Crit Care Med 2003;167:809-10.

12. Huebner RE, Schein MF, Bass JB Jr. The tuberculin skin test. Clin Infect Dis 1993;17:968-75.

13. Pai M, Riley LW, Colford JM Jr. Interferon-gamma assays in the immunodiagnosis of tuberculosis: a systematic review. Lancet Infect Dis 2004;4:761-76.

14. Page KR, Sifakis F, Montes de Oca R, et al. Improved adherence and less toxicity with rifampin vs isoniazid for treatment of latent tuberculosis: a retrospective study. Arch Intern Med 2006;166:1863-70.

15. Forget $\mathbf{E J}$, Menzies D. Adverse reactions to first-line antituberculosis drugs. Expert Opin Drug Saf 2006:5:231-49.

16. Yew WW, Leung CC. Antituberculosis drugs and hepatotoxicity. Respirology 2006;11:699-707.

17. Ponce de Leon D, Acevedo-Vasquez E, Sanchez-Torres A, et al. Attenuated response to purified protein derivative in patients with rheumatoid arthritis: study in a population with a high prevalence of tuberculosis. Ann Rheum Dis 2005;64:1360-1.

18. Mow WS, Abreu-Martin MT, Papadakis KA, et al. High incidence of anergy in inflammatory bowel disease patients limits the usefulness of PPD screening before infliximab therapy. Clin Gastroenterol Hepatol 2004;2:309-13.

19. Barnes PF. Diagnosing latent tuberculosis infection: the 100-year upgrade. Am J Respir Crit Care Med 2001;163:807-8.

20. Mariette $\mathbf{X}$, Salmon D. French guidelines for diagnosis and treating latent and active tuberculosis in patients with RA treated with TNF blockers. Ann Rheum Dis 2003;62:791.

21. Hamdi H, Mariette X, Godot V, et al. Inhibition of anti-tuberculosis T-lymphocyte function with tumour necrosis factor antagonists. Arthritis Res Ther 2006:8:R114.

22. Efthimiou P, Sood S. QuantiFERON TB Gold Test: the new standard for screening of latent tuberculosis in patients with rheumatoid arthritis? Ann Rheum Dis 2007:66:276

23. Martin J, Walsh C, Gibbs A, et al. Comparison of interferon (gamma) release assays and conventional screening tests before tumour necrosis factor $\{$ alpha\} blockade in patients with inflammatory arthritis. Ann Rheum Dis 2010;69:181-5.

24. Sellam J, Hamdi H, Roy C, et al. Comparison of in vitro-specific blood tests with tuberculin skin test for diagnosis of latent tuberculosis before anti-TNF therapy. Ann Rheum Dis 2007;66:1610-15.

25. Matulis G, Jüni P, Villiger PM, et al. Detection of latent tuberculosis in immunosuppressed patients with autoimmune diseases: performance of a Mycobacterium tuberculosis antigen-specific interferon gamma assay. Ann Rheum Dis 2008;67:84-90

26. Kleinert S, Kurzai 0, Elias J, et al. Comparison of two interferon-gamma release assays and tuberculin skin test for detecting latent tuberculosis in patients with immune-mediated inflammatory diseases. Ann Rheum Dis 2010;69:782-4.

27. Bartalesi F, Vicidomini S, Goletti D, et al. QuantiFERON-TB Gold and the TST are both useful for latent tuberculosis infection screening in autoimmune diseases. Eur Respir J 2009;33:586-93

28. Diel R, Loddenkemper R, Nienhaus A. Evidence-based comparison of commercial interferon-gamma release assays for detecting active TB: a metaanalysis. Chest 2010;137:952-68

29. Pai M, Zwerling A, Menzies D. Systematic review: T-cell-based assays for the diagnosis of latent tuberculosis infection: an update. Ann Intern Med 2008;149:177-84.

30. Sester M, Sotgiu G, Lange C, et al. Interferon-? release assays for the diagnosis of active tuberculosis: a systematic review and meta-analysis. Eur Respir $\mathrm{J}$ 2011;37:100-11.

31. Bocchino M, Matarese A, Bellofiore B, et al. Performance of two commercial blood IFN-gamma release assays for the detection of Mycobacterium tuberculosis infection in patient candidates for anti-TNF-alpha treatment. Eur J Clin Microbiol Infect Dis 2008;27:907-13.

32. Solovic I, Sester M, Gomez-Reino JJ, et al. The risk of tuberculosis related to tumour necrosis factor antagonist therapies: a TBNET consensus statement. Eur Respir J 2010;36:1185-206. 